\title{
Correlation Between Body Composition Analysis and Biochemical Nutritional Markers in Maintenance Hemodialysis Patients with Chronic Liver Disease
}

\author{
LUCIANA CARMEN NITOI ${ }^{*}$, VALERIU ARDELEANU², ANCA PANTEA STOIAN ${ }^{3 *}$, \\ LAVINIA ALEXANDRA MOROIANU ${ }^{1}$ \\ 1 „Dunarea de Jos” University, Faculty of Medicine and Pharmacy, Department of Clinical Medicine, Al. I. Cuza Str., 800010, \\ Galati, Romania \\ ${ }^{2}$ „Ovidius” University, Faculty of Medicine, 1 Universitatii Alley, 900470, Constanta, Romania \\ ",Carol Davila" University of Medicine and Pharmacy, Faculty of Medicine, Diabetes, Nutrition and Metabolic diseases \\ Department, 8 Eroii Sanitari Str., 050474, Bucharest, Romania
}

\begin{abstract}
Several approaches have been used to assess protein-energy wasting syndrome, such as clinical evaluation, biochemical nutritional markers, anthropometric measurements, but Bioelectrical Impedance Analysis (BIA) techniques hold a central place in clinical settings. The aim of this study is to report our clinical experience with BIA and the correlations between biochemical nutritional markers and BIA nutritional parameters in hemodialysis (HD) patients associating or free of chronic liver disease. This cross-sectional observational study included 69 HD patients divided into two groups: 33 with chronic liver disease (CLD+) versus 36 chronic liver disease-free (CLD-) from one HD unit in Romania. Serum albumin (SA), serum creatinine $(S C r)$ and $C$-reactive protein $(C R P)$ were obtained from the HD arterial line immediately before the HD session and by BIA the body composition including total body water (TBW), total body fat (TBF), lean fat free mass(LFFM), body muscular mass (BMM), malnutrition index and body protein reserve $(P R)$ were assessed. No significant differences between groups were found in BCM, BMM, PR and TBF ( $p=0.92, p=0.60, p=0.907$, and $p=0.634$, respectively). Malnutrition index had a significantly higher mean value in $H D-C L D(+)$ patients $(p=$ 0.00). HD-CLD(-) group showed a strong correlation between SA and SCr and BCM, BMM (kg), LFFM $(\mathrm{kg})$ and body $P R(\mathrm{~kg})(r=.48, r=.50, r=.44, r=.50$; resp. $r=.42, r=.40, r=.36, r=.42)$. In $H D-C L D(+)$ patients, a significant positive correlation was found between $S A$ and $S C r$ and LFFM and body PR $(r=.37, r=.35$; resp. $r=.44, r=.35)$. Discussion: BIA is one of the most accurate techniques for assessing nutritional status and should be regularly used in clinical practice along with biochemical nutritional markers in HD patients. Although the protein metabolism depends to a large extent on liver function, CLD cannot be considered as having a significant impact on nutritional status in HD patients.
\end{abstract}

Keywords: serum albumin, biochemical markers, bioelectrical impedance analysis

\section{Introduction}

Among the numerous complications of chronic kidney disease (CKD), progressive decline in body protein mass and energy reserves is one of the most typical and detrimental. This has been termed protein-energy wasting (PEW) [1]. The latest studies highlight the fact that PEW affects the prognosis of HD patients more than other comorbidities such as hyperphosphatemia, vascular calcification, or secondary hyperthyroidism [2]. PEW is considered an interconnected clinical, biochemical, and metabolic disorder. Assessment of nutritional status is, therefore, an integral part of nutritional management, so several biochemical nutritional markers, such as SA, SCr, and CRP have been used to assess the body protein stores. Of these, SA has been the most commonly used as it assesses both muscle and visceral protein status and is the standard recommended by KDOQI [3,4]. It is also the most inexpensive, available laboratory test and studied serum protein [5], and its concentration is influenced by hepatic synthesis, urinary losses, and dialysis process, but also by the presence of an acute or chronic

*email:luciananitoi@yahoo.com,ancastoian@yahoo.com 
inflammatory syndrome. CRP, an acute-phase protein, has been proposed as nutritionally-related biochemical marker. Besides being a marker of inflammation with pro-inflammatory properties, elevated plasma CRP was found to reflect chronic systemic inflammation in HD patients [6], but also was described as a sensitive and independent contributing factor for malnutrition [7]. SCr, is another good indicator of somatic protein stores, a declining predialysis level indicating a decrease of skeletal muscle mass [8]. Finally, it was found that there is no single ideal marker to assess nutritional status [9] in patients with CKD, in whom various metabolic alterations and other confounding factors, such as fluid overload, are common [10]. BIA, a technique recommended by the National Kidney Foundation's Kidney Disease Outcomes Quality Initiative, was proven to be safe and easy to use and was found to be an appropriate tool for the cross-sectional and follow-up assessment of nutritional status along with the biochemical markers of nutritional status in chronic HD patients. This is the first study on PEW in HD patients in Romania [11].

The aims of this study were:

1. To assess the nutritional status by BIA in HD patients, and to determine if there is a relationship between BIA nutritional parameters and the biochemical nutritional markers routinely used in this population;

2. To find if CLD has an impact on nutritional status in maintenance HD patients.

\section{Material and methods}

\subsection{Study design and patient characteristics}

We conducted an observational cross-sectional study of 69 hemodynamically stable patients (divided into two groups: 36 CLD-free and 33 CLD patients) on maintenance hemodialysis (MHD) (4 h, 3 times a week) for at least three months in a single dialysis unit, B. Braun Avitum Botosani, Romania, recruited in December 2015. Informed consent was obtained from all participants before enrolling in the study. The study was approved by the Hospital's Ethics Committee and by the Ethics Committee of the Iasi "Gr. T. Popa" University of Medicine and Pharmacy and was conducted in agreement with the Helsinki Declaration of Human Rights. The exclusion criteria were age under 18 years, current acute illnesses, mental illnesses, and refusal to participate in the study. Demographic characteristics, associated chronic medical conditions, biochemical and dialysis parameters were obtained from patients' medical records and from their primary nephrologist. Our CLD cases were HBV and HCV-related, compensated hemodynamically, and in metabolic stage.

\subsection{Biochemical assays}

Blood samples were obtained from the HD arterial line immediately before the HD session, after the patients had fasted overnight, but not necessarily on a midweek dialysis day. For the measurement of SA the quantitative immunoturbidimetiric method and for the measurement of SCr the enzymatic colorimetric Jaffe reaction were used. Standard laboratory methods were used for the measurements of serum hemoglobin, CRP, AST, ALT, and Kt/V urea.

\subsection{Bioelectrical Impedance Analysis of body composition}

The electrical properties of biological tissues are currently classified as active or passive response based on the source of electricity. Active response (bioelectricity) occurs when biological tissue provokes electricity from ionic activities inside cells, as in electrocardiograph signals from the heart and electroencephalograph signals from the brain. Passive response occurs when biological tissues are stimulated through an external electrical current source [12]. Bioimpedance or biological impedance is defined as the ability of biological tissue to impede electric current [13]. In our study, we used Body Composition Analyzer (Maltron Bio Scan 920-2, Medical Device Class IIA, UK), and assessments were performed in the supine position, with the use of an eight hand and feet tactile electrode system placed on 8 distinctive areas: one pair on lower arm, second above the cubital fossa and near to clavicle, the third one on lower leg, and the last one above the knee and the iliac crest. The single frequency 
bioimpedance analysis at $50 \mathrm{kHz}$ estimated the body compartments: total body water (TBW), extra- and intracellular water (ECW, ICW), body cell mass (BCM), total fat mass (TFM), fat free mass (FFM), protein mass and body muscle mass (BMM), directly measured in the first $30 \mathrm{~min}$ of the HD session.

\subsection{Statistical analysis}

The analysis was made with SPSS 16.0 and a $P$ value $<0.05$ was considered statistically significant. Baseline characteristics of the study sample, assessed by descriptive statistics, are presented as mean \pm standard deviation (SD) as appropriate. Pearson's correlation was used to assess the magnitude and direction of association between biological nutritional markers and BIA parameters. ANOVA and tstudent test were used to compare the quantitative nutritional BIA parameters and biohemical nutritional indicators between the groups and sexes.

\section{Results and discussions}

Because of the deterioration of nutrients in the storage and altered hepatic synthesis, hypoalbuminemia is commonly seen in HD patients with CLD [14]. Nutritional status assessment of MHD patients by biochemical nutritional markers and body composition revealed certain differences between the two patient groups (HD-CLD(-)/HD-CLD(+)) and between sexes. Our study subjects were relatively young, mean age was $50.68 \pm 9.66$ years and sex distribution was relatively balanced in both groups (Chi-square $=2.06, \mathrm{p}=0.00$ ). They received adequate dialysis treatment according to $\mathrm{KtV}$ cut-off recommended by K/DOQI [15]. So, 77.8\% of the CLD-free HD patients exceeded the Kt/V cut-off of 1.4 , compared to only half of $\mathrm{HD}-\mathrm{CLD}(+)$ patients (Chi-square $=4.18, \mathrm{p}=0.04)$ (Table 1). Mean SA in HD patients with CLD was slightly lower than in the control group $(\mathrm{t}=0.763, \mathrm{p}=0.448)$, and in women compared to males $(\mathrm{t}=0.482, \mathrm{p}=0.631$ ) (Table 1-3). More than half of HD patients with CLD did not reach the target of $4 \mathrm{~g} / \mathrm{dL}$ recommended by the international nutrition guidelines. This is probably due to 2 main reasons: (1) HD per se induces intense protein catabolism, event aggravated by the inflammatory status, anorexia, metabolic acidosis and inadequate protein intake, and (2) liver is the main site of albumin synthesis. It is important to note that our HD patients with CLD did not reach the stage of liver cirrhosis. It was found that renal failure per se does not produce a gross defect in the capacity of patients from having a normal rate of albumin syntesis [16]. There are some studies that showed the lack of prompt success of nutritional intervention in correcting hypoalbuminemia, suggesting that nonnutritional factors may contribute to or be responsible for reduced albumin concentration in a fraction of hypoalbuminemic dialysis patients [17]. The morphological and functional integrity of the liver is vital to human health in general as well as to patients with renal disease. The use of standard reference values for aminotransferases to help detect liver disease is less useful in patients on chronic HD, while liver biopsy may be helpful for assessing the activity and severity of liver disease, especially in chronic viral liver diseases [18]. This can explain why SA levels recorded in our CLD subject were lower despite the fact that mean aminotransferase levels were within normal range, and indeed lower than in the control group (Table 1). We can conclude that liver damage is an important factor negatively influencing the nutritional status as the liver is the main site of protein syntesis, but besides this there are many other factors that contribute to the development of malnutrition in end-stage renal disease. A diminished appetite is common in HD patients. Appetite is considered to be the gateway to the nutritional state [19]. Inadequate dialysis treatment can lead to uremic anorexia and poor nutritional intake in the presence of low appetite followed by the development of hypoalbuminemia, situation common in our HD-CLD $(+)$ group where only half of the subjects reached the Kt/V cutoff index.

In the CLD-free HD patients, a statistically significant correlation was found between SA and TBW (1), BCM, BMM (kg), LFFM (kg) and body PR (kg) ( $\mathrm{r}=.36, \mathrm{r}=.48, \mathrm{r}=.50, \mathrm{r}=.44, \mathrm{r}=.50$, respectively). In the group with CLD, SA also showed a strong relationship with LFFM and body PR ( $\mathrm{r}=.37$ and $\mathrm{r}=.35$, respectively) and a weak negative correlation with malnutrition index ( $\mathrm{r}=.15)$ (Table 3). 
Table 1. Comparative mean values of biochemical markers between both groups and sexes

\begin{tabular}{|c|c|c|c|c|c|c|c|c|}
\hline \multicolumn{2}{|c|}{$\begin{array}{c}\text { Biochemical nutritional markers } \\
\text { Mean } \pm \text { SD }\end{array}$} & Hemoglobin & SAlb & $\mathrm{SCr}$ & CRP & Kt/V uree & AST & ALT \\
\hline \multirow{3}{*}{$\begin{array}{c}\text { HD-CLD-vs } \\
\text { HD-CLD+ }\end{array}$} & HD-CLD- & $10.69 \pm 1.76$ & $4.05 \pm 0.25$ & $10.93 \pm 2.71$ & $2.44 \pm 1.79$ & $1.56 \pm 0.23$ & $27.31 \pm 21.97$ & $28.61 \pm 28.02$ \\
\hline & HD-CLD+ & $11.39 \pm 1.34$ & $3.99 \pm 0.32$ & $10.47 \pm 2.17$ & $8.76 \pm 5.82$ & $1.47 \pm 0.21$ & $25.61 \pm 17.77$ & $26.73 \pm 25.33$ \\
\hline & $P$ & 0.099 & 0.448 & 0.442 & 0.000 & 0.076 & 0.592 & 0.755 \\
\hline \multirow{3}{*}{$\begin{array}{l}\text { Sex MA vs. } \\
\text { FEM }\end{array}$} & MASC & $11.42 \pm 1.36$ & $4.03 \pm 0.29$ & $11.09 \pm 2.48$ & $5.95 \pm 5.71$ & 1.47 & $26.24 \pm 17.58$ & $31.83 \pm 31.73$ \\
\hline & FEM & $10.40 \pm 1.78$ & $4.00 \pm 0.27$ & $10.12 \pm 2.36$ & $4.70 \pm 4.46$ & $1.60 \pm 0.22$ & $26.89 \pm 23.50$ & $21.30 \pm 13.80$ \\
\hline & $P$ & 0.018 & 0.631 & 0.111 & 0.344 & 0.015 & 0.730 & 0.110 \\
\hline \multirow{3}{*}{$\begin{array}{l}\text { HD-CLD(-) } \\
\text { group }\end{array}$} & MASC & $11.05 \pm 1.39$ & $4.07 \pm 0.26$ & $11.25 \pm 2.76$ & $2.38 \pm 1.68$ & $1.51 \pm 0.21$ & $28.05 \pm 20.89$ & $35.00 \pm 35.15$ \\
\hline & FEM & $10.29 \pm 2.08$ & $4.01 \pm 0.24$ & $10.58 \pm 2.70$ & $2.50 \pm 1.96$ & $1.63 \pm 0.24$ & $26.47 \pm 23.73$ & $21.47 \pm 14.99$ \\
\hline & $P$ & 0.346 & 0.473 & 0.470 & 0.975 & 0.132 & 0.552 & 0.100 \\
\hline \multirow{3}{*}{$\begin{array}{c}\mathrm{HD}-\mathrm{CLD}(+) \\
\text { group }\end{array}$} & MASC & $11.73 \pm 1.28$ & $4.00 \pm 0.32$ & $10.97 \pm 2.27$ & $8.90 \pm 6.20$ & $1.43 \pm 0.21$ & $24.74 \pm 14.63$ & $29.22 \pm 29.15$ \\
\hline & FEM & $10.60 \pm 1.17$ & $3.98 \pm 0.33$ & $9.34 \pm 1.43$ & $8.44 \pm 5.11$ & $1.56 \pm 0.19$ & $27.60 \pm 24.36$ & $21.00 \pm 12.27$ \\
\hline & $p$ & 0.025 & 0.847 & 0.046 & 0.923 & 0.116 & 0.862 & 0.550 \\
\hline
\end{tabular}

HD-CLD(+)- hemodialysed patients with chronic liver disease; HD-CLD(-) - hemodialysed patients chronic liver disease free; CRP- Creactive protein; ALT- alanine aminotransferase; AST- aspartate aminotransferase; $p<0.05$ significant statistically;

SD-standard deviation.

BCM showed no significant differences between the groups $(\mathrm{p}=0.92)$, but significantly lower mean values in females compared to males $(\mathrm{p}=0.00)$ (Table 2). Malnutrition index had a significantly higher mean value in patients with CLD ( $\mathrm{p}=0.00)$ and in females $(\mathrm{p}=0.00)$ (Table 2). As to BMM, no significant differences were found between groups $(\mathrm{p}=0.60)$, but indeed men had greater muscle mass than women in the whole study group ( $\mathrm{p}=0.00$ ) and also separately (Table 2). The lowest BMM values were recorded in women with hepatic impairment $(23.11 \pm 2.69)$, and males with CLD also showed lower BMM values $(30.14 \pm 4.11)$ than those without CLD $(31.88 \pm 4.90)$. Body protein reserve assessed by BIA followed a similar trend. Again, the lowest PR levels were recorded in women with CLD (10.35 \pm 1.013$)$. Regarding TBF $(\mathrm{kg})$ no differences were found either between groups or sexes. On the other hand, LFFM $(\%)$ showed a statistically significant gender difference $(\mathrm{p}=0.00)$, to the detriment of women (Table 2). Statistically significant comparative data for the whole study sample and various biochemical and medical parameters are summarized in Tables 1-3.

Many factors influence SCr concentration including age, sex, race, dialysis dose, and residual renal function. It reflects in part the status of lean tissue, and to a lesser extent, recent dietary protein intake, serving as a non-specific indicator of protein status [20]. Long-term changes mirror alterations in lean tissue mass and probably decreased protein intake. $\mathrm{SCr}$ had a slightly higher mean value in the control group and in men but without statistically significant effect $(\mathrm{t}=0.77, \mathrm{p}=0.44 ; \mathrm{t}=1.61, \mathrm{p}=0.11$, respectively) (Table 1). Indeed, in CLD-free HD patients, $\mathrm{SCr}$ showed a direct and significant correlation with BCM, BMM, LFFM, and body PR ( $\mathrm{r}=.42, \mathrm{r}=.40, \mathrm{r}=.36, \mathrm{r}=.42$, respectively). Compared to the control group, in CLD+ HD patients, SCr also showed a significant correlation with LFFM and body PR $(\mathrm{r}=.44$ and $\mathrm{r}=.35$, respectively) (Table 3$)$. This study demonstrates that both biological nutritional markers, SA and SCr, show a strong relationship with BIA nutritional parameters (BMM, LFFM, PR and malnutrition index), which means that biochemical measures of nutritional status can reflect early alterations in nutrient supplies to tissues, more advanced changes in tissue components, and other alterations in metabolism [18].

Inflammation may itself promote anorexia, inadequate intake, and protein catabolism. It is wellknown the interaction between inflammation and protein wasting syndrome which gave rise to the concept of malnutrition-inflammation complex syndrome [21]. Marked SA declines are frequently due to inflammation [22], hypothesis confirmed by our study in the HD-CLD(+) group. Despite this, we found no correlation between inflammation and BIA parameters in the HD-CLD $(+)$ group. Since a single index of inflammation was available for the study patients, it was not possible to fully investigate the correlation between the inflammatory syndrome and BIA parameters. High CRP levels (Chi-square = $22.00, p=0.00$ ) were recorded in $5.6 \%$ of patients in the control group and $57.6 \%$ HD -CLD $(+)$ patients. Our study revealed a significant inverse correlation between serum CPR and TBF and LFFM $(\mathrm{r}=.65$ and $\mathrm{r}=.35$, respectively) in CLD-free HD patients (Table 3 ). 
Table 2. Comparative BIA parameters between groups and sexes

\begin{tabular}{|c|c|c|c|c|c|c|c|c|}
\hline \multirow{2}{*}{$\begin{array}{c}\text { BIA } \\
\text { parameters } \\
\text { (t student } \\
\text { test) }\end{array}$} & \multicolumn{2}{|c|}{$\begin{array}{l}\text { Group HD-CLD-vs. } \\
\text { group HD-CLD+ }\end{array}$} & \multicolumn{2}{|c|}{ Males vs. Females } & \multicolumn{2}{|c|}{$\begin{array}{c}\text { Group HDCLD- / males vs. } \\
\text { females }\end{array}$} & \multicolumn{2}{|c|}{$\begin{array}{c}\text { GroupHDCLD+/ males vs. } \\
\text { Females }\end{array}$} \\
\hline & $\begin{array}{l}\text { HD-CLD- } \\
n=36\end{array}$ & $\begin{array}{c}\text { HD-CLD+ } \\
n=33\end{array}$ & $\begin{array}{c}\text { Males }=\mathrm{n}=4 \\
2\end{array}$ & $\begin{array}{c}\text { Females }=\mathrm{n}=2 \\
7\end{array}$ & $\begin{array}{l}\text { Males }=\mathrm{n}=1 \\
9\end{array}$ & $\begin{array}{c}\text { Females }=\mathrm{n}=1 \\
7\end{array}$ & $\begin{array}{c}\text { Males }=n=2 \\
3\end{array}$ & $\begin{array}{c}\text { Females }=\mathrm{n}=1 \\
0\end{array}$ \\
\hline \multirow{2}{*}{$\begin{array}{c}\text { BCM - } \\
\text { Body Cell } \\
\text { Mass (kg) }\end{array}$} & $2.27 \pm 7.15$ & $32.13 \pm 5.18$ & $5.75 \pm 4.53$ & $24.46 \pm 4.95$ & $8.12 \pm 4.34$ & $24.33 \pm 4.32$ & $25.62 \pm 4.46$ & $24.68 \pm 6.13$ \\
\hline & \multicolumn{2}{|c|}{$\mathrm{t}=0.090 . \mathrm{p}=0.929$} & \multicolumn{2}{|c|}{$\mathrm{t}=5.832, \mathbf{p}=\mathbf{0 . 0 0 0}$} & \multicolumn{2}{|c|}{$\mathrm{t}=3.802, \mathbf{p}=\mathbf{0 . 0 0 1}$} & \multicolumn{2}{|c|}{$\mathrm{t}=5.096, \mathbf{p}=\mathbf{0 . 0 0 0}$} \\
\hline \multirow{2}{*}{$\begin{array}{l}\text { TBW - } \\
\text { Total Body } \\
\text { Water (l) }\end{array}$} & $42.47 \pm 9.40$ & $43.60 \pm 7.63$ & $46.78 \pm 6.17$ & $37.14 \pm 8.52$ & $47.91 \pm 6.94$ & $36.39 \pm 8.04$ & $45.85 \pm 5.44$ & $38.42 \pm 9.58$ \\
\hline & \multicolumn{2}{|c|}{$\mathrm{t}=-0.549, \mathrm{p}=0.585$} & \multicolumn{2}{|c|}{$\mathrm{t}=5.445, \mathbf{p}=\mathbf{0 . 0 0 0}$} & \multicolumn{2}{|c|}{$\mathrm{t}=4.611, \mathbf{p}=\mathbf{0 . 0 0 0}$} & \multicolumn{2}{|c|}{$\mathrm{t}=2.840, \mathbf{p}=\mathbf{0 . 0 0 8}$} \\
\hline \multirow{2}{*}{$\begin{array}{c}\text { TBW Total } \\
\text { Body } \\
\text { Water } \% \\
\end{array}$} & $55.59 \pm 8.52$ & $57.84 \pm 7.94$ & $8.05 \pm 7.68$ & $54.50 \pm 8.82$ & $56.51 \pm 8.88$ & $54.56 \pm 8.26$ & $59.33 \pm 6.46$ & $54.41 \pm 10.18$ \\
\hline & \multicolumn{2}{|c|}{$\mathrm{t}=-1.131, \mathrm{p}=0.262$} & \multicolumn{2}{|c|}{$\mathrm{t}=1.767, \mathrm{p}=0.082$} & \multicolumn{2}{|c|}{$\mathrm{t}=0.679, \mathrm{p}=0.502$} & \multicolumn{2}{|c|}{$\mathrm{t}=1.682, \mathrm{p}=0.103$} \\
\hline \multirow{2}{*}{$\begin{array}{c}\text { Malnutritio } \\
\mathrm{n} \text { index }\end{array}$} & $0.67 \pm 0.04$ & $0.78 \pm 0.06$ & $0.70 \pm 0.06$ & $0.76 \pm 0.08$ & $0.64 \pm 0.02$ & $0.70 \pm 0.03$ & $0.74 \pm 0.03$ & $0.86 \pm 0.05$ \\
\hline & \multicolumn{2}{|c|}{$\mathrm{t}=-8.035, \mathbf{p}=\mathbf{0 . 0 0 0}$} & \multicolumn{2}{|c|}{$\mathrm{t}=-3.434, \mathbf{p}=\mathbf{0 . 0 0 1}$} & \multicolumn{2}{|c|}{$\mathrm{t}=-7.087, \mathbf{p}=\mathbf{0 . 0 0 0}$} & \multicolumn{2}{|c|}{$t=-6.314, \mathbf{p}=\mathbf{0 . 0 0 0}$} \\
\hline \multirow{2}{*}{$\begin{array}{c}\text { ECW - } \\
\text { extra } \\
\text { cellular } \\
\text { water }(\%)\end{array}$} & $46.38 \pm 1.83$ & $46.6 \pm 1.92$ & $46.64 \pm 2.00$ & $46.28 \pm 1.64$ & $46.91 \pm 2.14$ & $45.79 \pm 1.23$ & $46.41 \pm 1.90$ & $47.12 \pm 1.96$ \\
\hline & \multicolumn{2}{|c|}{$\mathrm{t}=-0.542, \mathrm{p}=0.590$} & \multicolumn{2}{|c|}{$\mathrm{t}=0.765, \mathrm{p}=0.447$} & \multicolumn{2}{|c|}{$\mathrm{t}=1.879, \mathrm{p}=0.069$} & \multicolumn{2}{|c|}{$\mathrm{t}=-0.963, \mathrm{p}=0.343$} \\
\hline \multirow{2}{*}{$\begin{array}{c}\text { ECW - } \\
\text { extra } \\
\text { cellular } \\
\text { water (l) }\end{array}$} & $19.78 \pm 4.89$ & $19.78 \pm 3.47$ & $21.81 \pm 3.47$ & $16.72 \pm 3.40$ & $22.55 \pm 3.82$ & $16.68 \pm 4.06$ & $21.21 \pm 3.10$ & $16.77 \pm 2.05$ \\
\hline & $\mathrm{t}=-0.081$ & $p=0.936$ & $\mathrm{t}=5.996$ & $p=0.000$ & $\mathrm{t}=4.46$ & $p=0.000$ & $\mathrm{t}=4.12$ & $\mathbf{p}=$ \\
\hline ICW - & $23.37 \pm 7.02$ & $22.74 \pm 3.56$ & $25.5 \pm 5.4$ & $19.26 \pm 3.39$ & $26.74 \pm 7.45$ & $19.59 \pm 4.09$ & $24.49 \pm 2.57$ & $18.70 \pm 1.73$ \\
\hline $\begin{array}{l}\text { Intracelular } \\
\text { Water (1) }\end{array}$ & $\mathrm{t}=0.475$ & $p=0.637$ & $\mathrm{t}=5.35$ & $p=0.000$ & $\mathrm{t}=3.507$ & $p=0.001$ & $t=6.46$ & 000 \\
\hline $\mathrm{BMM}-$ & $28.70 \pm 6.07$ & $28.0 \pm 4.94$ & $.93 \pm 4.51$ & $24.38 \pm 4.55$ & $31.88 \pm 4.90$ & $25.14 \pm 5.29$ & $30.14 \pm 4.11$ & $23.11 \pm 2.69$ \\
\hline $\begin{array}{c}\text { body } \\
\text { muscle } \\
\text { mass }(\mathrm{kg})\end{array}$ & $t=0.516$ & $p=0.608$ & $t=5.853$ & $p=0.000$ & $\mathrm{t}=3.971$ & $p=0.000$ & $\mathrm{t}=4.94$ & 0 \\
\hline $\begin{array}{c}\text { TBF - } \\
\text { Total Body }\end{array}$ & $20.70 \pm 9.81$ & $\begin{array}{l}19.50 \pm 10.9 \\
9\end{array}$ & $19.89 \pm 8.92$ & $20.50 \pm 12.38$ & $21.52 \pm 9.62$ & $19.79 \pm 10.23$ & $18.55 \pm 8.28$ & $21.70 \pm 15.94$ \\
\hline Fat (kg) & $t=0$. & $p=0.634$ & $t=-0.23$ & $\mathrm{p}=0.815$ & $t=0.52$ & $p=0.604$ & $t=0.52$ & $p=0.604$ \\
\hline TBF - & $25.91 \pm 8.16$ & $24.34 \pm 8.94$ & $23.24 \pm 7.03$ & $28.15 \pm 9.82$ & $24.29 \pm 8.52$ & $27.71 \pm 7.10$ & $22.36 \pm 9.08$ & $28.90 \pm 11.46$ \\
\hline $\begin{array}{l}\text { Total Body } \\
\text { Fat }(\%)\end{array}$ & $t=0.759$ & $p=0.451$ & $\mathrm{t}=-2.422$ & $p=0.018$ & $t=-1.26$ & $\mathrm{p}=0.215$ & $\mathrm{t}=-2.02$ & $p=0.052$ \\
\hline LFFM - & $.17 \pm 7.76$ & $73.06 \pm 9.17$ & $5.60 \pm 6.85$ & $71.69 \pm 10.08$ & $9.91 \pm 3.28$ & $71.99 \pm 9.20$ & $73.88 \pm 7.84$ & $71.17 \pm 11.94$ \\
\hline $\begin{array}{l}\text { Lean Fat } \\
\text { Free Mass } \\
(\%)\end{array}$ & $\mathrm{t}=1.526$ & $p=0.132$ & $\mathrm{t}=2.413$ & $p=0.019$ & $\mathrm{t}=3.356$ & $p=0.003$ & $\mathrm{t}=0.77$ & $\mathrm{p}=0.444$ \\
\hline $\begin{array}{l}\text { LFFM - } \\
\text { Lean Fat }\end{array}$ & $\begin{array}{c}55.96 \pm 12.3 \\
1\end{array}$ & $55.88 \pm 8.93$ & $61.30 \pm 8.04$ & $47.55 \pm 9.14$ & $\begin{array}{l}62.88 \pm 10.8 \\
2\end{array}$ & $48.22 \pm 10.82$ & $60.00 \pm 6.95$ & $46.42 \pm 4.79$ \\
\hline $\begin{array}{l}\text { Free Mass } \\
(\mathrm{kg})\end{array}$ & $\mathrm{t}=0.029$ & 0.977 & $t=6.615$ & $=0.000$ & $\mathrm{t}=4.405$ & $=0.000$ & $=5.59$ & $=0.000$ \\
\hline PR- & $12.32 \pm 2.72$ & $12.39 \pm 1.95$ & $13.45 \pm 1.94$ & $10.65 \pm 1.96$ & $13.66 \pm 2.35$ & $10.83 \pm 2.36$ & $13.28 \pm 1.55$ & $10.35 \pm 1.01$ \\
\hline $\begin{array}{l}\text { Protein } \\
\text { Reserve of } \\
\text { the body } \\
(\mathrm{kg})\end{array}$ & $\mathrm{t}=-0.118$ & $\mathrm{p}=0.907$ & $\mathrm{t}=5.816$ & $p=0.000$ & $\mathrm{t}=3.58 \mathrm{~s}$ & $p=0.001$ & $\mathrm{t}=5.44$ & $p=0.000$ \\
\hline
\end{tabular}


Table 3. Pearson's correlation between BIA parameters and biochemical nutritional markers in both groups

\begin{tabular}{|c|c|c|c|c|c|c|c|c|c|c|c|c|c|c|c|}
\hline $\begin{array}{c}\text { Biochemical } \\
\text { mutritionsl } \\
\text { markers }\end{array}$ & Group & TBW (l) & $\begin{array}{l}\text { TBWW } \\
(96)\end{array}$ & ECW (I) & $\begin{array}{c}\mathrm{ECW} \\
(\mathrm{SG})\end{array}$ & ICW (l) & $\begin{array}{l}\text { ICWV } \\
\text { (96) }\end{array}$ & BCMI & MII & $\begin{array}{c}\text { BMMI } \\
(\mathrm{kg})\end{array}$ & TBF (kg) & $\begin{array}{l}\text { TBF } \\
\text { (96) }\end{array}$ & $\begin{array}{c}\text { LFFMI } \\
(\mathrm{kg})\end{array}$ & $\begin{array}{c}\text { LFFMI } \\
(96)\end{array}$ & $\begin{array}{c}\text { PR } \\
(\mathrm{Kg})\end{array}$ \\
\hline \multirow[t]{2}{*}{ SA } & $\begin{array}{l}\text { HD- } \\
\text { CLD. }\end{array}$ & $\begin{array}{l}R=361 \\
\mathbb{R}=130\end{array}$ & $\begin{array}{l}\mathrm{R}=.223 \\
\mathrm{R}^{2}=050\end{array}$ & $\begin{array}{l}\mathrm{R}=.096 \\
\mathrm{R}^{2}=.009\end{array}$ & $\begin{array}{l}\mathrm{R}=134 \\
\mathrm{R}=018\end{array}$ & $\begin{array}{l}R=408 \\
R=166\end{array}$ & $\begin{array}{l}R=113 \\
R=013\end{array}$ & $\begin{array}{r}\mathrm{R}=.482 \\
\mathrm{R}=.232\end{array}$ & $\begin{array}{l}R=001 \\
R=000\end{array}$ & $\begin{array}{l}R=504 \\
R=254\end{array}$ & $\begin{array}{l}R=.269 \\
R=073\end{array}$ & $\begin{array}{l}\mathrm{R}=.459 \\
\mathrm{R}=210\end{array}$ & $\begin{array}{l}R=.448 \\
\mathbb{R}=201\end{array}$ & $\begin{array}{l}R=0.052 \\
R=003\end{array}$ & $\begin{array}{l}R=500 \\
\mathbb{R}=250\end{array}$ \\
\hline & $\begin{array}{c}\mathrm{HD}- \\
\text { CID+ }\end{array}$ & $\begin{array}{l}R=188 \\
R=.035\end{array}$ & $\begin{array}{l}\mathrm{R}=130 \\
\mathrm{R}=017\end{array}$ & $\begin{array}{l}R=.184 \\
R=.034\end{array}$ & $\begin{array}{l}\mathrm{R}=272 \\
\mathrm{R}=074\end{array}$ & $\begin{array}{l}R=066 \\
\mathbb{R}=004\end{array}$ & $\begin{array}{l}R=025 \\
R=001\end{array}$ & $\begin{aligned} R & =.232 \\
\mathrm{R} & =.054\end{aligned}$ & $\begin{array}{l}R=155 \\
\mathbb{R}=050\end{array}$ & $\begin{array}{l}R=.223 \\
R=050\end{array}$ & $\begin{array}{l}R=033 \\
\mathbb{R}=001\end{array}$ & $\begin{array}{l}R=0.082 \\
R^{2}=007\end{array}$ & $\begin{array}{l}R=370 \\
R=137\end{array}$ & $\begin{aligned} R & =036 \\
R & =001\end{aligned}$ & $\begin{array}{l}R=350 \\
\mathbb{R}=1.123\end{array}$ \\
\hline \multirow[t]{2}{*}{$\mathrm{sCr}$} & $\begin{array}{l}\text { HD- } \\
\text { CLD. }\end{array}$ & $\begin{array}{l}R=001 \\
\mathbb{R}=000\end{array}$ & $\begin{array}{l}R=167 \\
\mathbb{R}=028\end{array}$ & $\begin{array}{l}R=083 \\
R=007\end{array}$ & $\begin{array}{l}\mathbb{R}=0.037 \\
\mathbb{R}=001\end{array}$ & $\begin{array}{l}\mathrm{R}=355 \\
\mathrm{R}=126\end{array}$ & $\begin{array}{l}R=126 \\
R=016\end{array}$ & $\begin{aligned} R & =.420 \\
\mathrm{R} & =177\end{aligned}$ & $\begin{array}{l}R=185 \\
\mathbb{R}=034\end{array}$ & $\begin{array}{l}\mathrm{R}=400 \\
\mathrm{R}=160\end{array}$ & $\begin{array}{l}R=181 \\
\mathbb{R}=033\end{array}$ & $\begin{array}{l}R=.269 \\
R=073\end{array}$ & $\begin{array}{l}\mathrm{R}=368 \\
\mathrm{R}=135\end{array}$ & $\begin{array}{l}\mathrm{R}=0003 \\
\mathrm{R}=.000\end{array}$ & $\begin{array}{l}\mathrm{R}=.423 \\
\mathrm{R}=179\end{array}$ \\
\hline & $\begin{array}{c}\text { HD- } \\
\text { CID+ }\end{array}$ & $\begin{array}{l}R=0886 \\
R=007\end{array}$ & $\begin{array}{l}\mathrm{R}=0.024 \\
\mathrm{R}=001\end{array}$ & $\begin{array}{l}\mathrm{R}=.210 \\
\mathrm{R}=044\end{array}$ & $\begin{array}{l}\mathrm{R}=302 \\
\mathrm{R}=091\end{array}$ & $\begin{array}{l}R=370 \\
\mathbb{R}=137\end{array}$ & $\begin{array}{l}\mathrm{R}=160 \\
\mathrm{R}=026\end{array}$ & $\begin{array}{l}\mathrm{R}=.237 \\
\mathrm{R}=.056\end{array}$ & $\begin{array}{l}R=.287 \\
\mathbb{R}=082\end{array}$ & $\begin{array}{l}\mathrm{R}=3 \mathrm{BS} \\
\mathrm{R}=14 \mathrm{~S}\end{array}$ & $\begin{array}{l}R=104 \\
\mathbb{R}=011\end{array}$ & $\begin{array}{l}R=009 \\
R=000\end{array}$ & $\begin{array}{l}R=328 \\
\mathbb{R}=107\end{array}$ & $\begin{array}{l}\mathrm{R}=0.082 \\
\mathrm{R}=.007\end{array}$ & $\begin{array}{l}R=.447 \\
R=200\end{array}$ \\
\hline \multirow[t]{2}{*}{ CRP } & $\begin{array}{l}\text { HD- } \\
\text { CLD. }\end{array}$ & $\begin{array}{l}R=131 \\
\mathbb{R}=017\end{array}$ & $\begin{array}{l}\mathrm{R}=.226 \\
\mathrm{R}=051\end{array}$ & $\begin{array}{l}R=015 \\
\mathbb{R}=000\end{array}$ & $\begin{array}{l}\mathrm{R}=323 \\
\mathrm{R}=104\end{array}$ & $\begin{array}{l}R=.293 \\
R^{2}=.086\end{array}$ & $\begin{array}{l}\mathrm{R}=291 \\
\mathrm{R}^{2}=085\end{array}$ & $\begin{array}{l}\mathrm{R}=.182 \\
\mathrm{R}=.033\end{array}$ & $\begin{array}{l}R=157 \\
R=025\end{array}$ & $\begin{array}{l}\mathrm{R}=216 \\
\mathrm{R}=047\end{array}$ & $\begin{array}{l}R=.271 \\
R=073\end{array}$ & $\begin{array}{l}\mathrm{R}=365 \\
\mathrm{R}=134\end{array}$ & $\begin{array}{l}R=.251 \\
R^{2}=.063\end{array}$ & $\begin{array}{l}\mathrm{R}=354 \\
\mathrm{R}=125\end{array}$ & $\begin{array}{l}R=.231 \\
\mathbb{R}^{2}=.053\end{array}$ \\
\hline & $\begin{array}{c}\text { HD- } \\
\text { CLD+ }\end{array}$ & $\begin{array}{l}R=155 \\
\mathbb{R}=024\end{array}$ & $\begin{array}{l}\mathrm{R}=174 \\
\mathrm{R}=030\end{array}$ & $\begin{aligned} R & =074 \\
\mathbb{R} & =006\end{aligned}$ & $\begin{array}{l}R=155 \\
\mathbb{R}=024\end{array}$ & $\begin{aligned} \mathbb{R} & =019 \\
\mathbb{R} & =0000\end{aligned}$ & $\begin{array}{l}R=080 \\
\mathbb{R}=006\end{array}$ & $\begin{aligned} \mathrm{R} & =.243 \\
\mathrm{R} & =059\end{aligned}$ & $\begin{array}{l}R=056 \\
\mathbb{R}=003\end{array}$ & $\begin{array}{l}\mathbb{R}=281 \\
\mathbb{R}=079\end{array}$ & $\begin{array}{l}\mathrm{R}=139 \\
\mathrm{R}=019\end{array}$ & $\begin{array}{l}\mathbb{R}=194 \\
\mathbb{R}=038\end{array}$ & $\begin{aligned} R & =0008 \\
\mathbb{R} & =000\end{aligned}$ & $\begin{aligned} \mathbb{R} & =025 \\
R & =001\end{aligned}$ & $\begin{array}{l}R=083 \\
R=007\end{array}$ \\
\hline
\end{tabular}

HD-CLD(+)- hemodialysis patients with chronic liver disease; HD-CLD(-) - hemodialysis patients chronic liver disease free; BIA-bioimpendance analysis; TBW - Total Body Water; ECW - Extracellular water; ICW -Intracelular Water; BCM - Body Cell Mass; MI- malnutrition index; BMM -Muscle mass; TBF - Total Body Fat; LFFM - Lean Fat Free Mass; PR-Protein Reserve of the body; SA- serum albumin, Scr - serum creatinine. CRP -C-reactive protein.

\section{Conclusions}

Biochemical nutritional markers are still playing a key role in nutritional status assessment. SA, which is still considered a reliable malnutrition index, by its decline in plasma demonstrated a relatively late manifestation of malnutrition since albumin has a long half-life and hepatic synthetic reserve is very large. Although the protein metabolism depends to a large extent upon liver function, chronic liver disease cannot be incriminated as having a significant detrimental impact on nutritional status in HD patients. In conclusion, to illustrate an adequate nutritional status assessment, besides clinical evaluation and biochemical markers, bioelectrical analysis of human body is supported by our study results. The limitations of this study, namely small sample size, cross-sectional design, and subject recruitment from a single dialysis unit, strengthen the idea of a longer survey of the studied population to better acknowledge the prognostic value of tested variables.

\section{Abreviations}

ALT - alanine aminotransferase
AST- aspartat aminotransferase
BCM- body cell mass
BIA- bioimpendance analysis of body composition
BMM- body muscular mass
CKD- chronic kidney disease
CLD- chronic liver disease
CRP- C reactive protein
ECW- extracellular water
FFM- fat free mass
HBV- hepatitis B virus
HCV- hepatitis C virus

HD- hemodialysis

ICW- intracellular water

KDQOI- kidney disease quality of life initiative

$\mathrm{Kt} / \mathrm{V}$ - marker measuring hemodialysis adequacy

LFFM- lean fat free mass

PEW- protein energy wasting syndrom

PR- body protein reserve

SA- serum albumin

SCr- serum creatinine

TBW- total body water

TBF- total body fat

TFM- total fat mass

\section{References}

1.FOUQUE, D., KALANTAR-ZADEH, K., KOPPLE, J., et al, A proposed nomenclature and diagnostic criteria for protein energy wasting in acute and chronic kidney disease, Kidney Int., 73(4), 2008, 391398.

2.INOUE, A., ISHIKAWA, E., SHIRAI, Y., MURATA, T., MIKI, C., HAMADA, Y., Effects of Protein-Energy Wasting (PEW) and hyperphosphatemia on the prognosis in Japanese maintenance hemodialysis patients: A five-year follow-up observational study, Clin Nutr. ESPEN, 36, 2020, 134138.

3.BERNARDO, A.P, FONSECA, I., RODRIGUES, A., et al., Overweight rather than malnutrition is widely prevalent in peritoneal dialysis patients, Adv Perit Dial., 25, 2009, 119-124. 
4.FOUQUE, D., VENNEGOOR, M., TER WEE, P., et al., EBPG guideline on nutrition, Nephrol Dial Transplan., 22(2), 2007, ii45-87.

5.SUHAIR, A.K.A., YOUSIF B.Y., "Effect of Nutritional Counseling in the Form of Individualized Meal Plan on Serum Albumin Level among Hemodialysis Patients", EC Nutrition 5.4, 2016, 1181-1187. 6.KAUR, S., SINGH, N.P., JAIN, A.K., THAKUR, A., Serum C-reactive protein and leptin for assessment of nutritional status in patients on maintenance hemodialysis, Indian J Nephrol., 2012; 22(6), 419-423.

7.CARRERO, J.J, QURESHI, A.R., AXELSSON, J., et al. Comparison of nutritional and inflammatory markers in dialysis patients with reduced appetite. Am J Clin Nutr., 85(3), 2007, 695-701.

8.AZAR, R., AL-MOUBARAK, I., BARSUMAU, J., et al., Assessment and follow-up of nutritional status in hemodialysis patients, Néphrologie \& Thérapeutique, 5(5), 2009, S317-322.

9.GAMA-AXELSSON, T., HEIMBÜRGER, O., STENVINKEL, P., et al., Serum albumin as predictor of nutritional status in patients with ESRD, Clin J Am Soc Nephrol., 7(9), 2012, 1446-1453.

10.ALI, G., FATEMEH, H., MAJID, K., SOUDABEH, S., The prevalence of malnutrition in hemodiaşysis patients, J Renal Inj Prev., 9(2), 2020, 1-6.

11.VEISA, G., DONCIU, M.D., SEGAL, L., et al., Albumin as a prognostic factor for malnutrition and inflammation in chronic kidney disease, Rev. Chim., 67(1), 2016, 103-105.

12.KYLE, U.G., BOSAEUS, I., DE LORENZO, A.D., et al., Bioelectrical impedance analysis Part I: Review of principles and methods, Clin. Nutr., 23 (5), 2004, 1226-1243.

13.MARTINSEN, O.G., GRIMNES, S., Bioimpedance and Bioelectricity Basics. Academic Press, Waltham, MA, USA: 2011.

14.KORETZ, R.L., The evidence for use of nutritional support in liver disease, Current Opinion in Gastroenterology, 30(2), 2014, 208-214.

15.NKF-K/DOQI. Clinical Practice Guidelines for Hemodialysis Adequacy: update 2000. Am J Kidney Dis., 37(1), 2001, S7-S64.

16.TOJO, A., KINUGASA, S., Mechanisms of glomerular albumin filtration and tubular reabsorption. Int J Nephrol., 2012: 481520.

17.DONCIU, M.D., FLOREA, L., MITITIUC, L.I., POPESCU, E.R., CHIRITA, R., Biochemical and Bioimpedance Correlation with Quality of Life and Illness Perception in Hemodialysis Patients., Rev.Chim., 70(2), 2019, 679-684.

18.HRSTIĆ, I., OSTOJIĆ, R., Chronic liver disease in chronic kidney disease, Acta Med Croatica, 65(4), 2011, 349-353.

19.KALANTAR-ZADEH K., KOPPLE, J.D, Nutritional Management of Maintenance Hemodialysis Patients, Nutritional manaement in renal disease_3 $3^{\text {rd }}$ Edition, 32, 2013, 503-538.

20.KOPPLE, J.D., National kidney foundation K/DOQI clinical practice guidelines for nutrition in chronic renal failure, Am J Kidney Dis., 37(1), 2001, S66-70.

21.FERNANDEZ REY, L., RODAS VISIEDO, L., RODRIGUEZ LOPEZ, F., et al., Utility of malnutrition inflammation score (MIS) to predict mortality in hemodialysis patients, Clinical Nutrition, 37(1), 2018, S207.

22.ALVES, F.C., SUN, J., QURESHI, A.R., et al., The higher mortality associated with low serum albumin is dependent on systemic inflammation in end-stage kidney disease, PLoS ONE, 13(1), 2018, $\mathrm{e} 0190410$.

$\overline{\text { Manuscript received: } 8.02 .2020}$ 